\title{
To the solution of the problem of bending of a cylindrical shell by the boundary elements method
}

\author{
Mykola Surianinov ${ }^{1, *}$, and Yurii Krutii ${ }^{1}$ \\ ${ }^{1}$ Odessa State Academy of Civil Engineering and Architecture, Didrikson str. 4, Odessa, 65029, \\ Ukraine
}

\begin{abstract}
The solution of the problem of the long cylindrical shell bending by a numerical and analytical boundary elements method is considered. The method is based on the analytical construction of a fundamental system of solutions and Green's functions for the differential equation of the problem under consideration. This paper is devoted to the determination of these expressions. The semi-moment theory of the cylindrical shell calculation, proposed by V.Z. Vlasov, which for the problem under consideration leads to one eighth-order partial differential equation is used. The problem of the bending of a cylindrical shell is twodimensional, and in the numerical and analytical boundary elements method, plates and shells are considered as generalized one-dimensional modules, so the variational method of Kantorovich-Vlasov was applied to this equation to obtain an ordinary differential equation of the eighth order. Sixty-four expressions of all the fundamental functions of the problem are constructed, as well as an analytic expression for the Green's function, which makes it possible to construct a load vector (without any restrictions on the nature of its application), and then proceed to the solution of boundary-value problems for the bending of long cylindrical shells under various boundary conditions.
\end{abstract}

\section{Introduction}

The stress state of a cylindrical shell and the corresponding theory of its calculation depend substantially on the length of the shell. In the case of a long shell supported along curvilinear edges and loaded with an arbitrary smoothly varying load, its stress state is close to the one of the beam. With an uneven distribution of the load along the shell and along the cross-section, this approach gives incorrect results, and we must take into account the deformation of the contour. This also applies to the case of securing the longitudinal edges of the shell.

V.Z. Vlasov proposed a theory of long cylindrical shells calculation, which was called a semi-moment theory $[1,2]$. According to this model the shell consists of an infinite number of transversal curved elementary strips, joined connected by a system of rods with hinged

\footnotetext{
*Corresponding author: sng@ogasa.org.ua
} 
joints (Fig. 1). Each such rod can transmit only normal $N_{1}$ and shear $S$ forces. The bending moment in the longitudinal direction, the transverse force and the torque can be neglected, so the state vector $\overline{\mathbf{P}}$ of such a shell is represented in the form

$$
\overline{\mathbf{P}}^{\mathbf{T}}=\left\|W, W^{\prime}, M, Q, S, N, U, V\right\| .
$$

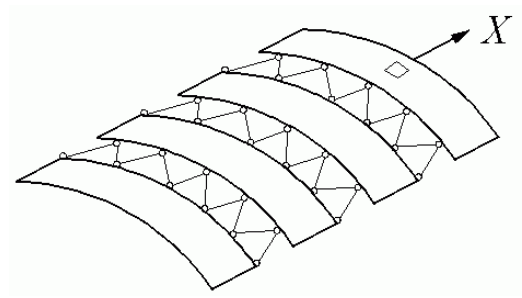

Fig. 1. Model of V.Z. Vlasov.

In total, in the state vector (1) there are 4 forces and 4 displacements (Fig. 2).

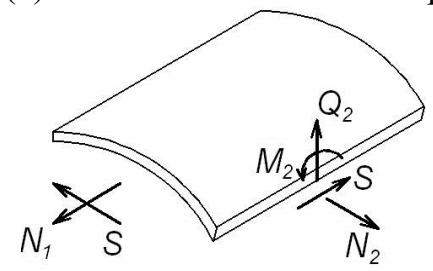

Fig. 2. Forces and displacements in shell.

In view of what has been said, all the equations of the general theory of shells are simplified [3-5], and as a result we get the basic resolving equation of the problem

$$
\left(\frac{\partial^{8}}{\partial \theta^{8}}+2 \frac{\partial^{6}}{\partial \theta^{6}}+\frac{\partial^{4}}{\partial \theta^{4}}\right) F+\frac{12 R^{2}}{h^{2}} \frac{\partial^{4} F}{\partial \beta^{4}}=0,
$$

where $F-$ new function (analog of stress function in the theory of plates), through which all the parameters of the state of the shell are expressed, $\theta-$ dimensionless coordinate (Fig. 3):

$$
\beta=x / R ; \quad \theta=s / R .
$$

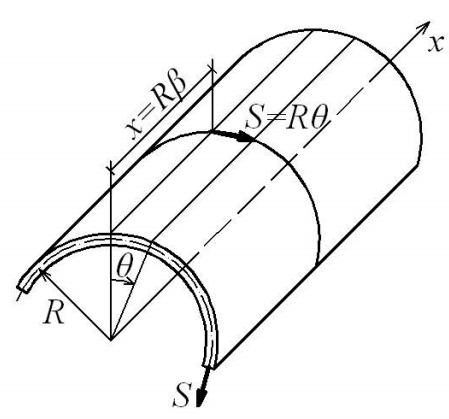

Fig. 3. The coordinate system used. 


\section{Results}

\subsection{Construction of the system of fundamental functions}

To solve the problem, we will use the numerical and analytical boundary elements method (NA BEM) [6-9]. The method is based on the analytical construction of a fundamental system of solutions and Green's functions for the differential equation of the problem under consideration. The boundary conditions, or the conditions of contact between modules (individual elements of the system) are taken into account by numerically solving a small system of linear algebraic equations.

The algorithm of the method can be determined by transformation according to the scheme:

$$
\overrightarrow{\mathbf{Y}}(l)=\overline{\mathbf{A}}(l) \vec{X}(0)+\overrightarrow{\mathbf{B}}(l) \rightarrow \overline{\mathbf{A}}(l) \overrightarrow{\mathbf{X}}(0)-\overrightarrow{\mathbf{Y}}(l)=-\overrightarrow{\mathbf{B}}(l) \rightarrow \overline{\mathbf{A}}_{*}(l) \overrightarrow{\mathbf{X}}_{*}(0, l)=-\overrightarrow{\mathbf{B}}(l),
$$

where the final boundary parameters of the matrix $\overrightarrow{\mathbf{Y}}$ are transferred to the place of zero vector parameters $\overrightarrow{\mathbf{X}}$. Moreover, these vectors are supplemented by equations of equilibrium and compatibility of displacements of nodal points and boundary conditions.

The purpose of this paper is to construct analytical expressions for the matrix $\overline{\mathbf{A}}$ and the Green's function, which is used in the formation of the load vector $\overrightarrow{\mathbf{B}}$.

The problem of a cylindrical shell bending is two-dimensional, but plates and shells are considered as generalized one-dimensional modules in the NA BEM, therefore, we applied Kantorovich-Vlasov's variational method to equation (2), resulting in a differential equation with constant coefficients:

$$
Y^{V 111}(\theta)+2 Y^{V 1}(\theta)+Y^{1 V}(\theta)+\frac{12 R^{2} \lambda^{4}}{h^{2}} Y(\theta)=0
$$

Here $\lambda-$ a certain parameter related to the frequency of natural oscillations; in the shell problem $\lambda=\frac{R m}{l}$, where $h$ and $l-$ thickness and length of shell; $R-$ shell radius; $m$ - the characteristic number determined when setting the boundary conditions.

Characteristic equation for (3) has a form [10]

$$
t^{8}+2 t^{6}+t^{4}+k=0
$$

where $k=\frac{12 R^{2} \lambda^{4}}{h^{2}}$.

Characteristic equation (4) has 8 roots:

$$
t_{1-4}= \pm \alpha_{1} \pm i \beta_{1} ; t_{5-8}= \pm \alpha_{2} \pm i \beta_{2} .
$$

We write the function $Y(\theta)$ in the form

$$
Y(\theta)=C_{1} \Phi_{1}+C_{2} \Phi_{2}+C_{3} \Phi_{3}+C_{4} \Phi_{4}+C_{5} \Phi_{5}+C_{6} \Phi_{6}+C_{7} \Phi_{7}+C_{8} \Phi_{8}
$$

where $\Phi_{1}-\Phi_{8}-$ hyperbolic-trigonometric functions: 


$$
\begin{cases}\Phi_{1}=\operatorname{ch} \alpha_{1} y \sin \beta_{1} y ; & \Phi_{2}=\operatorname{ch} \alpha_{1} y \cos \beta_{1} y \\ \Phi_{3}=\operatorname{sh} \alpha_{1} y \cos \beta_{1} y ; & \Phi_{4}=\operatorname{sh} \alpha_{1} y \sin \beta_{1} y \\ \Phi_{5}=\operatorname{ch} \alpha_{2} y \sin \beta_{2} y ; & \Phi_{6}=\operatorname{ch} \alpha_{2} y \cos \beta_{2} y \\ \Phi_{7}=\operatorname{sh} \alpha_{2} y \cos \beta_{2} y ; & \Phi_{8}=\operatorname{sh} \alpha_{2} y \sin \beta_{2} y\end{cases}
$$

Constants $C_{1}-C_{8}$ we determine from relations

$$
\bar{\Phi}_{i}(0) \vec{C}_{i}=\vec{P}_{i}(0), \quad i=1,2, \ldots, 8 .
$$

Expanding (8), we have eight algebraic equations that form two independent systems with respect to sets of constants with even and odd indices. In the coefficients of systems, functions $\Phi_{1}-\Phi_{8}$ and their derivatives up to the seventh order inclusive are used, therefore we introduce additional notation, with allowance for which the systems of equations are written in the form

$$
\begin{gathered}
\left\{\begin{array}{l}
H_{21} C_{1}+H_{23} C_{3}+H_{25} C_{5}+H_{27} C_{7}=W_{0}^{\prime} ; \\
H_{41} C_{1}+H_{43} C_{3}+H_{45} C_{5}+H_{47} C_{7}=Q_{0} ; \\
H_{51} C_{1}+H_{53} C_{3}+H_{55} C_{5}+H_{57} C_{7}=S_{0} ; \\
H_{81} C_{1}+H_{83} C_{3}+H_{85} C_{5}+H_{87} C_{7}=V_{0},
\end{array}\right. \\
\left\{\begin{array}{l}
H_{12} C_{2}+H_{14} C_{4}+H_{16} C_{6}+H_{18} C_{8}=W_{0} ; \\
H_{32} C_{2}+H_{34} C_{4}+H_{36} C_{6}+H_{38} C_{8}=M_{0} ; \\
H_{62} C_{2}+H_{64} C_{4}+H_{66} C_{6}+H_{68} C_{8}=N_{0} ; \\
H_{72} C_{2}+H_{74} C_{4}+H_{76} C_{6}+H_{78} C_{8}=U_{0},
\end{array}\right.
\end{gathered}
$$

where

$$
\begin{gathered}
H_{12}=\left(\alpha_{1}^{4}-6 \alpha_{1}^{2} \beta_{1}^{2}+\beta_{1}^{4}\right) ; H_{14}=4 \alpha_{1} \beta_{1}\left(\alpha_{1}^{2}-\beta_{1}^{2}\right) ; \\
H_{16}=\left(\alpha_{2}^{4}-6 \alpha_{2}^{2} \beta_{2}^{2}+\beta_{2}^{4}\right) ; H_{18}=4 \alpha_{2} \beta_{2}\left(\alpha_{2}^{2}-\beta_{2}^{2}\right) ; \\
H_{21}=\beta_{1}\left(5 \alpha_{1}^{4}-10 \alpha_{1}^{2} \beta_{1}^{2}+\beta_{1}^{4}\right) ; H_{23}=\alpha_{1}\left(\alpha_{1}^{4}-10 \alpha_{1}^{2} \beta_{1}^{2}+5 \beta_{1}^{4}\right) ; \\
H_{25}=\beta_{2}\left(5 \alpha_{2}^{4}-10 \alpha_{2}^{2} \beta_{2}^{2}+\beta_{2}^{4}\right) ; H_{27}=\alpha_{2}\left(\alpha_{2}^{4}-10 \alpha_{2}^{2} \beta_{2}^{2}+5 \beta_{2}^{4}\right) ; \\
H_{32}=-\frac{D}{R^{2}}\left(\alpha_{1}^{6}+\alpha_{1}^{4}-15 \alpha_{1}^{4} \beta_{1}^{2}-6 \alpha_{1}^{2} \beta_{1}^{2}+15 \alpha_{1}^{2} \beta_{1}^{4}+\beta_{1}^{4}-\beta_{1}^{6}\right) ; \\
H_{34}=-\frac{D}{R^{2}} \cdot 2 \alpha_{1} \beta_{1}\left(3 \alpha_{1}^{4}-10 \alpha_{1}^{2} \beta_{1}^{2}+3 \beta_{1}^{4}+2 \alpha_{1}^{2}-2 \beta_{1}^{2}\right) ; \\
H_{36}=-\frac{D}{R^{2}}\left(\alpha_{2}^{6}+\alpha_{2}^{4}-15 \alpha_{2}^{4} \beta_{2}^{2}-6 \alpha_{2}^{2} \beta_{2}^{2}+15 \alpha_{2}^{2} \beta_{2}^{4}+\beta_{2}^{4}-\beta_{2}^{6}\right) ;
\end{gathered}
$$




$$
\begin{aligned}
& H_{38}=-\frac{D}{R^{2}} 2 \alpha_{2} \beta_{2}\left(3 \alpha_{2}^{4}-10 \alpha_{2}^{2} \beta_{2}^{2}+3 \beta_{2}^{4}+2 \alpha_{2}^{2}-2 \beta_{2}^{2}\right) \\
& H_{41}=\frac{D}{R^{3}} \cdot \beta_{1}\left(7 \alpha_{1}^{6}-35 \alpha_{1}^{4} \beta_{1}^{2}+21 \alpha_{1}^{2} \beta_{1}^{4}-\beta_{1}^{6}+5 \alpha_{1}^{4}-10 \alpha_{1}^{2} \beta_{1}^{2}+\beta_{1}^{4}\right) \\
& H_{43}=\frac{D}{R^{3}} \cdot \alpha_{1}\left(\alpha_{1}^{6}-21 \alpha_{1}^{4} \beta_{1}^{2}+35 \alpha_{1}^{2} \beta_{1}^{4}-7 \beta_{1}^{6}+\alpha_{1}^{4}-10 \alpha_{1}^{2} \beta_{1}^{2}+5 \beta_{1}^{4}\right) \\
& H_{45}=\frac{D}{R^{3}} \cdot \beta_{2}\left(7 \alpha_{2}^{6}-35 \alpha_{2}^{4} \beta_{2}^{2}+21 \alpha_{2}^{2} \beta_{2}^{4}-\beta_{2}^{6}+5 \alpha_{2}^{4}-10 \alpha_{2}^{2} \beta_{2}^{2}+\beta_{2}^{4}\right) \\
& H_{47}=\frac{D}{R^{3}} \cdot \alpha_{2}\left(\alpha_{2}^{6}-21 \alpha_{2}^{4} \beta_{2}^{2}+35 \alpha_{2}^{2} \beta_{2}^{4}-7 \beta_{2}^{6}+\alpha_{2}^{4}-10 \alpha_{2}^{2} \beta_{2}^{2}+5 \beta_{2}^{4}\right) \\
& H_{51}=\beta_{1} \frac{E h}{R} ; H_{53}=\alpha_{1} \frac{E h}{R} ; H_{55}=\beta_{2} \frac{E h}{R} ; H_{57}=\alpha_{2} \frac{E h}{R} \\
& H_{62}=-\frac{E h}{R}\left[a+\alpha_{1}^{4}-6 \alpha_{1}^{2} \beta_{1}^{2}+\beta_{1}^{4}+b\left(\alpha_{1}^{6}-15 \alpha_{1}^{4} \beta_{1}^{2}+15 \alpha_{1}^{2} \beta_{1}^{4}-\beta_{1}^{6}\right)\right] \\
& H_{64}=-\frac{E h}{R}\left[4 \alpha_{1} \beta_{1}\left(\alpha_{1}^{2}-\beta_{1}^{2}\right)+2 b \alpha_{1} \beta_{1}\left(3 \alpha_{1}^{4}-10 \alpha_{1}^{2} \beta_{1}^{2}+3 \beta_{1}^{4}\right)\right] \\
& H_{66}=-\frac{E h}{R}\left[a+\alpha_{2}^{4}-6 \alpha_{2}^{2} \beta_{2}^{2}+\beta_{2}^{4}+b\left(\alpha_{2}^{6}-15 \alpha_{2}^{4} \beta_{2}^{2}+15 \alpha_{2}^{2} \beta_{2}^{4}-\beta_{2}^{6}\right)\right] \\
& H_{68}=-\frac{E h}{R}\left[4 \alpha_{2} \beta_{2}\left(\alpha_{2}^{2}-\beta_{2}^{2}\right)+2 b \alpha_{2} \beta_{2}\left(3 \alpha_{2}^{4}-10 \alpha_{2}^{2} \beta_{2}^{2}+3 \beta_{2}^{4}\right)\right] ; \\
& H_{72}=-\left(\alpha_{1}^{2}-\beta_{1}^{2}\right) ; H_{74}=-2 \alpha_{1} \beta_{1} ; H_{76}=-\left(\alpha_{2}^{2}-\beta_{2}^{2}\right) ; H_{78}=-2 \alpha_{2} \beta_{2} ; \\
& H_{81}=\beta_{1}\left(3 \alpha_{1}^{2}-\beta_{1}^{2}\right) ; H_{83}=\alpha_{1}\left(\alpha_{1}^{2}-3 \beta_{1}^{2}\right) ; H_{85}=\beta_{2}\left(3 \alpha_{2}^{2}-\beta_{2}^{2}\right) ; \\
& H_{87}=\alpha_{2}\left(\alpha_{2}^{2}-3 \beta_{2}^{2}\right) \text {. }
\end{aligned}
$$

Coefficients $H_{i j}$ of systems (9), (10) are arithmetic operators that are easily programmed and calculated, but the right-hand parts of these systems are parameters of the shell state vector. In accordance with the NA BEM algorithm [3], the constants must be expressed in a general form as a function of the parameters of the state vector, which will allow us to obtain analytic expressions for the fundamental functions.

This is achieved by solving the systems (9) and (10) by the determinants method. Consider, for example, the solution of system (9).

We assume that the principal determinant of this system is computed (this can be done in any corresponding mathematical program) i.e. is a known number, which we denote as $\Delta_{\text {odd }}$. 
The determinants $\Delta_{C_{1}}, \Delta_{C_{3}}, \Delta_{C_{5}}, \Delta_{C_{7}}$, can also be calculated by expanding them along the parameter column of the right-hand side of (9).

The solution of the system (9) takes the form

$$
\left\{\begin{array}{l}
C_{1}=\frac{1}{\Delta_{\text {odd }}}\left(\Delta_{W_{0}^{\prime}}^{(1)} W_{0}^{\prime}-\Delta_{Q_{0}}^{(1)} Q_{0}+\Delta_{S_{0}}^{(1)} S_{0}-\Delta_{V_{0}}^{(1)} V_{0}\right) \\
C_{3}=\frac{-1}{\Delta_{\text {odd }}}\left(\Delta_{W_{0}^{\prime}}^{(3)} W_{0}^{\prime}-\Delta_{Q_{0}}^{(3)} Q_{0}+\Delta_{S_{0}}^{(3)} S_{0}-\Delta_{V_{0}}^{(3)} V_{0}\right) \\
C_{5}=\frac{1}{\Delta_{o d d}}\left(\Delta_{W_{0}^{\prime}}^{(5)} W_{0}^{\prime}-\Delta_{Q_{0}}^{(5)} Q_{0}+\Delta_{S_{0}}^{(5)} S_{0}-\Delta_{V_{0}}^{(5)} V_{0}\right) \\
C_{7}=\frac{-1}{\Delta_{o d d}}\left(\Delta_{W_{0}^{\prime}}^{(7)} W_{0}^{\prime}-\Delta_{Q_{0}}^{(7)} Q_{0}+\Delta_{S_{0}}^{(7)} S_{0}-\Delta_{V_{0}}^{(7)} V_{0}\right)
\end{array}\right.
$$

Similarly, we approach the definition of a set of constants $C_{2}, C_{4}, C_{6}, C_{8}$ :

$$
\left\{\begin{array}{l}
C_{2}=\frac{1}{\Delta_{\text {even }}}\left(\Delta_{W_{0}}^{(2)} W_{0}-\Delta_{M_{0}}^{(2)} M_{0}+\Delta_{N_{0}}^{(2)} N_{0}-\Delta_{U_{0}}^{(2)} U_{0}\right) \\
C_{4}=\frac{-1}{\Delta_{\text {even }}}\left(\Delta_{W_{0}}^{(4)} W_{0}-\Delta_{M_{0}}^{(4)} M_{0}+\Delta_{N_{0}}^{(4)} N_{0}-\Delta_{U_{0}}^{(4)} U_{0}\right) \\
C_{6}=\frac{1}{\Delta_{\text {even }}}\left(\Delta_{W_{0}}^{(6)} W_{0}-\Delta_{M_{0}}^{(6)} M_{0}+\Delta_{N_{0}}^{(6)} N_{0}-\Delta_{U_{0}}^{(6)} U_{0}\right) ; \\
C_{8}=\frac{-1}{\Delta_{\text {even }}}\left(\Delta_{W_{0}}^{(8)} W_{0}-\Delta_{M_{0}}^{(8)} M_{0}+\Delta_{N_{0}}^{(8)} N_{0}-\Delta_{U_{0}}^{(8)} U_{0}\right)
\end{array}\right.
$$

We proceed to the definition of fundamental orthonormal functions.

$$
W=A_{11} W_{0}+A_{12} W_{0}^{\prime}+A_{13} M_{0}+A_{14} Q_{0}+A_{15} S_{0}+A_{16} N_{0}+A_{17} U_{0}+A_{18} V_{0} .
$$

On the other hand

$$
\begin{aligned}
& W=Y^{I V}=C_{1} \Phi_{1}^{I V}+C_{2} \Phi_{2}^{I V}+C_{3} \Phi_{3}^{I V}+C_{4} \Phi_{4}^{I V}+C_{5} \Phi_{5}^{I V}+ \\
& +C_{6} \Phi_{6}^{I V}+C_{7} \Phi_{7}^{I V}+C_{8} \Phi_{8}^{I V} .
\end{aligned}
$$

We substitute the values of the constants (11), (12) into expression (14) and regroup it with respect to the parameters of the state vector. Taking into account (13), the coefficients at these parameters will form the first eight fundamental functions:

$$
\begin{aligned}
& A_{11}=\frac{1}{\Delta_{\text {even }}}\left[\left(H_{12} \Phi_{2}-H_{14} \Phi_{4}\right) \Delta_{W_{0}}^{(2)}-\left(H_{12} \Phi_{4}+H_{14} \Phi_{2}\right) \Delta_{W_{0}}^{(4)}+\right. \\
& \left.+\left(H_{16} \Phi_{6}-H_{18} \Phi_{8}\right) \Delta_{W_{0}}^{(6)}-\left(H_{16} \Phi_{8}+H_{18} \Phi_{6}\right) \Delta_{W_{0}}^{(8)}\right]
\end{aligned}
$$




$$
\begin{aligned}
& A_{12}=\frac{1}{\Delta_{\text {odd }}}\left[\left(H_{12} \Phi_{1}+H_{14} \Phi_{3}\right) \Delta_{W_{0}^{\prime}}^{(1)}-\left(H_{12} \Phi_{3}-H_{14} \Phi_{1}\right) \Delta_{W_{0}^{\prime}}^{(3)}+\right. \\
& \left.+\left(H_{16} \Phi_{5}+H_{18} \Phi_{7}\right) \Delta_{W_{0}^{\prime}}^{(5)}-\left(H_{16} \Phi_{7}-H_{18} \Phi_{5}\right) \Delta_{W_{0}^{\prime}}^{(7)}\right] \\
& A_{13}=\frac{1}{\Delta_{\text {even }}}\left[-\left(H_{12} \Phi_{2}-H_{14} \Phi_{4}\right) \Delta_{M_{0}}^{(2)}+\left(H_{12} \Phi_{4}+H_{14} \Phi_{2}\right) \Delta_{M_{0}}^{(4)}-\right. \\
& \left.-\left(H_{16} \Phi_{6}-H_{18} \Phi_{8}\right) \Delta_{M_{0}}^{(6)}+\left(H_{16} \Phi_{8}+H_{18} \Phi_{6}\right) \Delta_{M_{0}}^{(8)}\right] ; \\
& A_{14}=\frac{1}{\Delta_{\text {odd }}}\left[-\left(H_{12} \Phi_{1}+H_{14} \Phi_{3}\right) \Delta_{Q_{0}}^{(1)}+\left(H_{12} \Phi_{3}-H_{14} \Phi_{1}\right) \Delta_{Q_{0}}^{(3)}-\right. \\
& \left.-\left(H_{16} \Phi_{5}+H_{18} \Phi_{7}\right) \Delta_{Q_{0}}^{(5)}+\left(H_{16} \Phi_{7}-H_{18} \Phi_{5}\right) \Delta_{Q_{0}}^{(7)}\right] ; \\
& A_{15}=\frac{1}{\Delta_{\text {odd }}}\left[\left(H_{12} \Phi_{1}+H_{14} \Phi_{3}\right) \Delta_{S_{0}}^{(1)}-\left(H_{12} \Phi_{3}-H_{14} \Phi_{1}\right) \Delta_{S_{0}}^{(3)}+\right. \\
& \left.+\left(H_{16} \Phi_{5}+H_{18} \Phi_{7}\right) \Delta_{S_{0}}^{(5)}-\left(H_{16} \Phi_{7}-H_{18} \Phi_{5}\right) \Delta_{S_{0}}^{(7)}\right] ; \\
& A_{16}=\frac{1}{\Delta_{\text {even }}}\left[\left(H_{12} \Phi_{2}-H_{14} \Phi_{4}\right) \Delta_{N_{0}}^{(2)}-\left(H_{12} \Phi_{4}+H_{14} \Phi_{2}\right) \Delta_{N_{0}}^{(4)}+\right. \\
& \left.+\left(H_{16} \Phi_{6}-H_{18} \Phi_{8}\right) \Delta_{N_{0}}^{(6)}-\left(H_{16} \Phi_{8}+H_{18} \Phi_{6}\right) \Delta_{N_{0}}^{(8)}\right] ; \\
& A_{17}=\frac{1}{\Delta_{\text {even }}}\left[-\left(H_{12} \Phi_{2}-H_{14} \Phi_{4}\right) \Delta_{U_{0}}^{(2)}+\left(H_{12} \Phi_{4}+H_{14} \Phi_{2}\right) \Delta_{U_{0}}^{(4)}-\right. \\
& \left.-\left(H_{16} \Phi_{6}-H_{18} \Phi_{8}\right) \Delta_{U_{0}}^{(6)}+\left(H_{16} \Phi_{8}+H_{18} \Phi_{6}\right) \Delta_{U_{0}}^{(8)}\right] \\
& \left.\left.\Delta_{\text {odd }}+H_{18} \Phi_{7}\right) \Delta_{V_{0}}^{(5)}+\left(H_{16} \Phi_{7}-H_{18} \Phi_{5}\right) \Delta_{V_{0}}^{(7)}\right] .
\end{aligned}
$$

The expressions for the remaining 56 fundamental functions are defined in a similar way.

\subsection{The construction of the Green's function}

One of the main operations for the implementation of the algorithm of NA BEM with respect to the problem of a long cylindrical shell bending is the formation of the vector of the right-hand sides (the load vector) of equation

$$
\overline{\mathbf{A}}_{*}(l) \cdot \overrightarrow{\mathbf{X}}(0, l)=-\overrightarrow{\mathbf{B}}(l),
$$


where $\overline{\mathbf{A}}_{*}(l)$ - a transformed matrix of orthonormal fundamental functions; $\overrightarrow{\mathbf{X}}(0, l)-$ vector of unknown initial and final parameters; $\overrightarrow{\mathbf{B}}(l)$ - vector of load elements [8, 9].

The construction of the vector of cargo elements involves the definition of the Green's function and its seven derivatives, since the stress-strain state of a long cylindrical shell is characterized by eight parameters when it is bent.

As is known [10], the mechanical mea 'ning of the Green's function is that this function determines the magnitude of the displacement from the unit concentrated impact. Taking into account the vector of the shell state parameters, this means that under the action of normal and tangential loads, the Green's function has the form

$$
G(y-\xi)=A_{14}(y-\xi)+A_{18}(y-\xi),
$$

where expressions $A_{14}(y-\xi)$ и $A_{18}(y-\xi)$ coincide with the expressions for the fundamental functions $A_{14}$ and $A_{18}$ obtained earlier, but from argument $(y-\xi)$.

Under real operating conditions, tangential loads on the shell are rare, and then will be equality

$$
G(y-\xi)=A_{14}(y-\xi)
$$

This assumption is confirmed by all our previous studies $[8,9]$, but we need to verify its validity with respect to a long cylindrical shell.

In accordance with the well-known algorithm for constructing the Green's function [8, 10], it can be represented in the form

$$
G(y, \xi)=Y(y, \xi) H(y-\xi),
$$

where $Y(y, \xi)$ - a particular solution of the differential equation of problem; $H(y-\xi)$ - Heaviside function.

For the Green's function, inequality $y>\xi$ performs, therefore $H(y-\xi)$ in (18) can be omitted.

A particular solution is formed in the form

$$
Y(y, \xi)=C_{1}(\xi) y_{1}(y)+C_{2}(\xi) y_{2}(y)+\ldots+C_{8}(\xi) y_{8}(y) .
$$

Constants $C_{i}(\xi)(i=1,2, \ldots, 8)$ are determined from condition

$$
\left|\begin{array}{crrr}
y_{1}(\xi) & y_{2}(\xi) & \ldots \ldots . & y_{8}(\xi) \\
y_{1}^{\prime}(\xi) & y_{2}^{\prime}(\xi) & \ldots \ldots & y_{8}^{\prime}(\xi) \\
\ldots \ldots \ldots \ldots \ldots \ldots \ldots \ldots \ldots \ldots \ldots \ldots \ldots \ldots . \\
y_{1}^{\text {III }}(\xi) & y_{2}^{\text {VII }}(\xi) & \ldots \ldots . & y_{8}^{\text {VII }}(\xi)
\end{array}\right|\left|\begin{array}{c}
C_{1} \\
C_{2} \\
\vdots \\
C_{8}
\end{array}\right|=\left|\begin{array}{c}
0 \\
0 \\
\vdots \\
1 / a_{0}
\end{array}\right|,
$$

where

$$
\left\{\begin{array}{l}
y_{1}=\operatorname{ch} \alpha_{1} \xi \sin \beta_{1} \xi ; \quad y_{2}=\operatorname{ch} \alpha_{1} \xi \cos \beta_{1} \xi ; \quad y_{3}=\operatorname{sh} \alpha_{1} \xi \cos \beta_{1} \xi \\
y_{4}=\operatorname{sh} \alpha_{1} \xi \sin \beta_{1} \xi ; \quad y_{5}=\operatorname{ch} \alpha_{2} \xi \sin \beta_{2} \xi ; \quad y_{6}=\operatorname{ch} \alpha_{2} \xi \cos \beta_{2} \xi \\
y_{7}=\operatorname{sh} \alpha_{2} \xi \cos \beta_{2} \xi ; \quad y_{8}=\operatorname{sh} \alpha_{2} \xi \sin \beta_{2} \xi .
\end{array}\right.
$$


Here $\alpha_{1}, \alpha_{2}, \beta_{1}, \beta_{2}-$ the coefficients of the roots of the characteristic equation corresponding to the differential equation of the shell bending [1].

The determinant of the system (20) is the Wronskian determinant, and is not zero for $y \in\left(0, y_{\text {boundary }}\right)$, therefore (20) has a unique solution. Coefficient $a_{0}$ (coefficient at the highest degree of the derivative in the differential equation of the shell bending) is equal to one, so we obtain a system of linear algebraic equations:

$$
\left\{\begin{array}{l}
y_{1} C_{1}+y_{2} C_{2}+\ldots+y_{8} C_{8}=0 \\
y_{1}^{\prime} C_{1}+y_{2}^{\prime} C_{2}+\ldots+y_{8}^{\prime} C_{8}=0 \\
\ldots \ldots \ldots \ldots \ldots \ldots \ldots \ldots \ldots \ldots \ldots \ldots \ldots \ldots \\
y_{1}^{V I I} C_{1}+y_{2}^{V I I} C_{2}+\ldots+y_{8}^{V I I} C_{8}=1 .
\end{array}\right.
$$

The derivatives of the functions (21) that enter into equations (22) can be expressed in terms of the functions $y_{i}$. The corresponding expressions are given in [8]. Denoting this coefficients by $H_{i j}(i, j=1,2, \ldots 8)$, we obtain a system of algebraic equations of the form

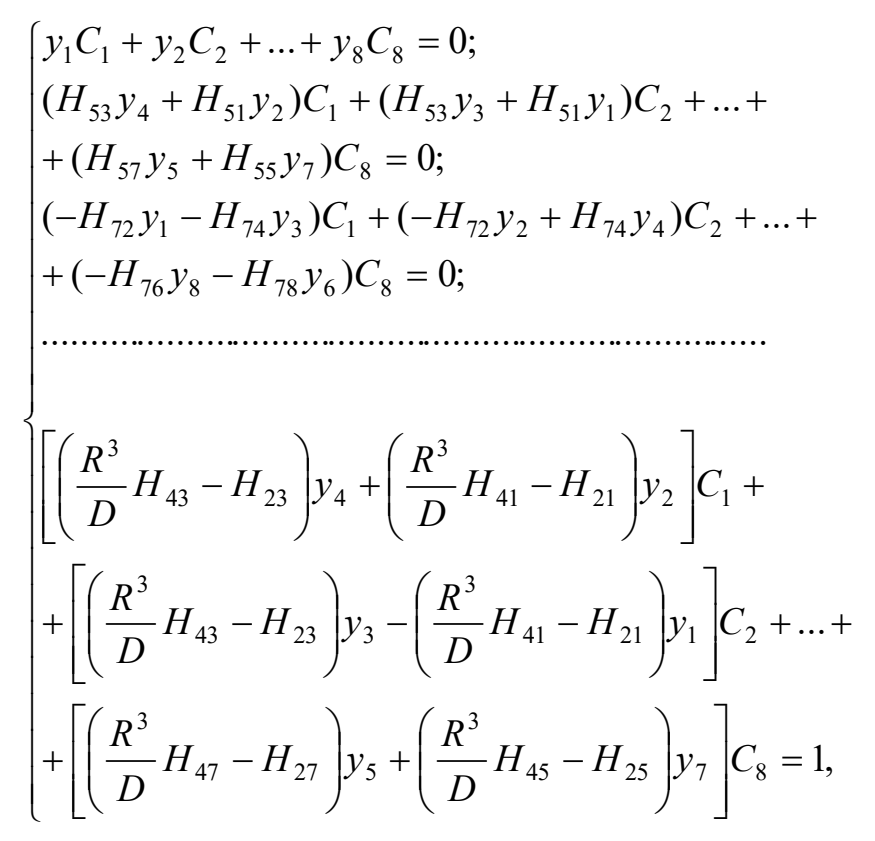

where $D=\frac{E h^{3}}{12}-$ cylindrical stiffness; $h$ and $R-$ thickness and radius of the shell.

Solving the system (23) by the Gauss method (note that in this case this process leads to rather cumbersome intermediate calculations), we determine the constants $C_{i}(\xi)(i=1,2, \ldots 8)$.

Finally, for the Green's function we obtain expression 


$$
\begin{aligned}
& G(y, \xi)=Y(y, \xi) H(y-\xi)=C_{1}(\xi) y_{1}(y)+C_{2}(\xi) y_{2}(y)+C_{8}(\xi) y_{8}(y)= \\
& =\frac{1}{\Delta_{\text {odd }}}\left[-\left(H_{12} y_{1}+H_{14} y_{3}\right) \Delta_{Q_{0}}^{(1)}+\left(H_{12} y_{3}-H_{14} y_{1}\right) \Delta_{Q_{0}}^{(3)}-\right. \\
& \left.-\left(H_{16} y_{5}+H_{18} y_{7}\right) \Delta_{Q_{0}}^{(5)}+\left(H_{16} y_{7}-H_{18} y_{5}\right) \Delta_{Q_{0}}^{(7)}\right],
\end{aligned}
$$

where through $\Delta_{Q_{0}}^{(1)}, \Delta_{Q_{0}}^{(3)}, \Delta_{Q_{0}}^{(5)}, \Delta_{Q_{0}}^{(7)}$ the determinants are denoted:

$$
\begin{gathered}
\Delta_{Q_{0}}^{(1)}=\left|\begin{array}{lll}
H_{23} & H_{25} & H_{27} \\
H_{53} & H_{55} & H_{57} \\
H_{83} & H_{85} & H_{87}
\end{array}\right| ; \Delta_{Q_{0}}^{(3)}=\left|\begin{array}{lll}
H_{21} & H_{25} & H_{27} \\
H_{51} & H_{55} & H_{57} \\
H_{81} & H_{85} & H_{87}
\end{array}\right| ; \\
\Delta_{Q_{0}}^{(5)}=\left|\begin{array}{lll}
H_{21} & H_{23} & H_{27} \\
H_{51} & H_{53} & H_{57} \\
H_{81} & H_{83} & H_{87}
\end{array}\right| ; \quad \Delta_{Q_{0}}^{(7)}=\left|\begin{array}{lll}
H_{21} & H_{23} & H_{25} \\
H_{51} & H_{53} & H_{55} \\
H_{81} & H_{83} & H_{85}
\end{array}\right| .
\end{gathered}
$$

A comparison of expression (24) with the analytical expressions obtained earlier for orthonormal fundamental functions confirms the assumption made at first, namely:

$$
G(y-\xi)=A_{14}(y-\xi) .
$$

It can be verified that the constructed function $G(y, \xi)$ has all the properties characteristic of the Green's function:

a) $G(y, \xi)=0$ at $y<\xi$;

b) $G(y, \xi)$ as function of $y$ at fixed $\xi$ in the interval $\left(0, y_{\text {boundary }}\right)$, excluding point $y=\xi$, satisfy the differential equation of problem;

c) $G(y, \xi)$ and its derivatives by $y$ up to $n$-th order for $y \in\left(0, y_{\text {boundary }}\right)$, excluding point $y=\xi$, in which the derivatives with respect to $y$ are continuous only up to $(n-2)$ order, and $(n-1)$ the derivative has a discontinuity of the first kind with a jump

$$
\left.\frac{d^{(n-1)} G(y, \xi)}{d y^{(n-1)}}\right|_{y=\xi+0}-\left.\frac{d^{(n-1)} G(y, \xi)}{d y^{(n-1)}}\right|_{y=\xi-0}=\frac{1}{a_{0}} ;
$$

d) at $y=\xi$,

$$
G(\xi, \xi)=G^{\prime}(\xi, \xi)=\ldots=G^{(n-2)}(\xi, \xi)=0 ; \quad G^{(n-1)}(\xi, \xi)=\frac{1}{a_{0}}=1
$$

e) $G(y, \xi)$ for the differential equation of the problem (and this is a differential equation with constant coefficients) depends only on the difference $(y-\xi)$. 


\section{Conclusions}

Thus, the basic analytical apparatus for solving the problem of the bending of a long cylindrical shell by a numerical and analytical boundary elements method is constructed. 64 expressions for all the fundamental functions of the problem are obtained, as well as an analytic expression for the Green's function, which makes it possible to construct a load vector (without any restrictions on the nature of its application), and then proceed to the solution of boundary-value problems for the bending of long cylindrical shells under various boundary conditions.

\section{References}

1. V.Z. Vlasov, General theory of shells and its application in technics (State Publishing House of Technical and Theoretical Literature, Moscow, 1948)

2. N.V. Kolkunov, Basics of elastic shells calculations (High School, Moskow, 1972)

3. S.P. Timoshenko, S. Woinowsky-Krieger, Plastiny i obolochki (Science, Moscow, 1966)

4. E. Reissner, On the foundations of Generalized linear shell theory (Copenhagen, 1967)

5. P.M. Naghdi, Fl. Han. Der Ph. Bd., VI, 425-640, (1972)

6. S.L. Crouch, A. M. Starfield, Boundary Element Methods in Solid Mechanics: With Applications in Rock Mechanics and Geological Engineering (George Allen \& Unwin, Crows Nest, 1983)

7. C.A. Brebbia, Recent Advances in Boundary Element Methods (Pentech Press, London, 1978)

8. A.F. Daschenko, L.V. Kolomiets, V.F. Orobey, M.H. Surianinov, Numerical and analytical method of boundary elements (VMV, Odesa, 2010)

9. V.F. Orobey, M.H. Surianinov, Praktworkshop on solving boundary value problems of mechanics (Astroprint, Odesa, 2011)

10. A.F. Bermant, Mathematical Analysis Course (State Publishing House of Technical and Theoretical Literature t, Moscow, 1953) 